\title{
Arborescences
}

Revue d'études françaises

\section{"Qualche novità litteraria ": la correspondance entre Jacob Spon (1647-1685) et Antonio Magliabechi (1633-1714)}

\section{Yves Moreau}

Numéro 9, décembre 2019

La lettre érudite. Nouvelles recherches sur la communication savante à l'époque moderne (XVI ${ }^{\mathrm{e}}$-XVIII ${ }^{\mathrm{e}}$ siècles)

URI : https://id.erudit.org/iderudit/1068276ar

DOI : https://doi.org/10.7202/1068276ar

Aller au sommaire du numéro

Éditeur(s)

Département d'études françaises, Université de Toronto

ISSN

1925-5357 (numérique)

Découvrir la revue

Citer cet article

Moreau, Y. (2019). « Qualche novità litteraria » : la correspondance entre Jacob Spon (1647-1685) et Antonio Magliabechi (1633-1714). Arborescences, (9), 81-94. https://doi.org/10.7202/1068276ar
Résumé de l'article

Cet article donne un aperçu de la manière dont sont partagées et reçues les informations scientifiques et littéraires contenues dans les 40 lettres de la correspondance échangée par le médecin et antiquaire lyonnais Jacob Spon et le bibliothécaire florentin Antonio Magliabechi entre 1675 et 1685. Si elles ne jouissent pas du même statut dans la République des Lettres, ces deux figures intellectuelles mobilisent leurs compétences pour favoriser leurs activités respectives autour des savoirs. Mais derrière ces échanges de services et de connaissances voulus par les règles de sociabilité savante, on identifie des processus de publicisation et de légitimation de leur position intellectuelle au sein de la République des Lettres.
Tous droits réservés @ C Département d'études françaises, Université de Toronto, 2020
Cedocument est protégé par la loi sur le droit d'auteur. L'utilisation des services d’Érudit (y compris la reproduction) est assujettie à sa politique d'utilisation que vous pouvez consulter en ligne.

https://apropos.erudit.org/fr/usagers/politique-dutilisation/ 


\section{SOMMAIRE}

1 Sébastien Drouin, Université de Toronto

Camelia Sararu, Université de Toronto

Introduction

10 Myron McShane, Centre d'études sur la Réforme et la Renaissance, Université de Toronto Une lettre de Jean Dorat sur l'œuvre de Nonnos

31 Benoît Autiquet, Université de Bâle

Ce que la lettre familière fait au discours médical.

Une lecture de la lettre XIX, 16 des Lettres de Pasquier (1619)

49 Guillaume Bazière, Université Paris Nanterre

Présence érudite et savoirs politiques dans la correspondance du Grand Condé

68 Vanezia Pârlea, Université de Bucarest

Lettres d'Orient : échanges épistolaires en contexte interculturel dans les Mémoires du chevalier d'Arvieux

81 Yves Moreau, Université de Lyon

"Qualche novità litteraria»: la correspondance

entre Jacob Spon (1647-1685) et Antonio Magliabechi (1633-1714)

95 Corinne Marchal, Université de Franche-Comté

La dynamique de la circulation des savoirs et de leurs matériaux dans les échanges épistolaires entre Jean-Baptiste Boisot et Paul Pellisson-Fontanier (1674-1693)

106 Mathilde Chollet, Le Mans Université

Un «gai savoir»: stratégies du rire dans les lettres

$d$ 'une érudite des Lumières 


\title{
"Qualche novità litteraria»: la correspondance entre Jacob Spon (1647-1685) et Antonio Magliabechi (1633-1714)
}

\author{
Yves Moreau, Université de Lyon
}

\section{Résumé}

Cet article donne un aperçu de la manière dont sont partagées et reçues les informations scientifiques et littéraires contenues dans les 40 lettres de la correspondance échangée par le médecin et antiquaire lyonnais Jacob Spon et le bibliothécaire florentin Antonio Magliabechi entre 1675 et 1685 . Si elles ne jouissent pas du même statut dans la République des Lettres, ces deux figures intellectuelles mobilisent leurs compétences pour favoriser leurs activités respectives autour des savoirs. Mais derrière ces échanges de services et de connaissances voulus par les règles de sociabilité savante, on identifie des processus de publicisation et de légitimation de leur position intellectuelle au sein de la République des Lettres.

Dans la seconde moitié du XVII ${ }^{\mathrm{e}}$ siècle, la correspondance manuscrite est le moyen privilégié pour la transmission des savoirs entre les membres de la République des Lettres, ce vaste "méta-réseau» de savants où s'élabore collectivement le Savoir. Mais malgré les discours d'universalité et d'égalité avancés pour décrire cette communauté, il existe pourtant, sur le plan géographique comme humain, des polarités. Les grands centres urbains européens concentrent savants, imprimeurs et libraires et monopolisent la production des Savoirs. Ils établissent une hiérarchie spatiale et sociale et polarisent de facto les échanges épistolaires, humains et commerciaux au sein de la République des Lettres. Cet article explore la relation épistolaire nouée entre le médecin lyonnais Jacob Spon et le bibliothécaire florentin Antonio Magliabechi. La quarantaine de lettres recensée, jamais publiée ni étudiée, donne à voir les dynamiques à l'œuvre dans les relations interpersonnelles au sein de la République des Lettres. Les deux savants ne jouissent pas de la même réputation ni du même statut social. Antonio Magliabechi, bibliothécaire du grand-duc de Toscane, est une des grandes figures de l'espace savant européen. Jacob Spon est un médecin et antiquaire reconnu pour ses compétences, habitant Lyon, une ville de foire et d'imprimerie de taille importante, mais à l'écart des grands circuits d'échanges intellectuels. Le partage d'informations, de services et d'ouvrages constitue l'essentiel du contenu de ces lettres, mais derrière ces rapports, on devine le jeu des motivations personnelles animant ces correspondants.

\section{Débuts et pérennisation d'une relation épistolaire érudite}

\subsection{La rencontre entre Jacob Spon et Antonio Magliabechi}

L'échange épistolaire entre Jacob Spon et Antonio Magliabechi est à l'initiative du jeune savant lyonnais qui séjourne en Italie en 1675. Médecin de formation, Spon s'intéresse aux vestiges matériels de l'Antiquité gréco-romaine: médailles, inscriptions, bas-reliefs (Étienne et Mossière 1993). Il recense et déchiffre les inscriptions de sa ville natale, Lyon, et publie ses travaux en 1673 dans un ouvrage intitulé Recherche des antiquités et curiosités de la ville de Lyon. Désireux de parfaire ses connaissances, il entreprend alors un voyage au-delà des Alpes (Rebaudo 1998). Le jeune médecin quitte Lyon vers le 15 octobre 1674 avec l'objectif de collecter des inscriptions antiques afin de donner un supplément 
aux Inscriptiones antique totius orbis romani (1602) de Jan Gruter, ouvrage épigraphique de référence à l'époque. Son voyage va le mener au-delà de la Péninsule italienne. Il s'embarque à Venise avec des compagnons anglais en juin 1675, visite la côte dalmate, puis se rend à Délos et Constantinople, passe par Bursa et rejoint Smyrne et Éphèse: puis, après un détour par Zante, il débarque à Lépante, fait étape à Delphes et à Thèbes et visite Athènes. Il retrouve Venise en mai 1676 et aperçoit enfin Lyon au mois d'août 1676 après un périple de 22 mois. Il tire de ce long voyage un ouvrage, Voyage d'Italie, de Dalmatie, de Grèce et du Levant (1678), achevé d'imprimer en novembre 1677.

La rencontre avec Antonio Magliabechi a lieu en mai 1675 à Florence dans le cadre de son itinéraire italien. Après avoir passé cinq mois à arpenter Rome, Jacob Spon fait une halte dans la capitale toscane avant de rejoindre Venise. Il se distingue dès son arrivée par le déchiffrement d'inscriptions antiques exposées dans la galerie des Offices. Il rencontre le cardinal Léopold de Médicis (1617-1675), qui lui présente personnellement ses collections d'antiquités (Spon, Voyage I: 64). Il fait la connaissance d'Antonio Magliabechi lors d'une visite des palais médicéens. Le bibliothécaire jouit déjà d'une grande réputation dans toute l'Europe pour ses connaissances bibliographiques. Il ouvre au visiteur les portes des bibliothèques grand-ducales du palazzo Pitti et de la Laurenziana. "La Bibliothèque du Palais [ducal] est bien fournie, mais les manuscrits qu'on tient à S. Laurens me plaisent encore plus ", écrit Spon dans la relation de son voyage (Voyage, I: 64-65). L'antiquaire est impressionné par la quantité de manuscrits rares que renferme la Laurenziana, en particulier en médecine. Il peut, grâce à la générosité de Magliabechi, recopier une quinzaine d'entre eux. On ignore si le bibliothécaire a accueilli le jeune savant lyonnais dans son "musée», autrement dit sa maison, qui abritait l'une des plus importantes bibliothèques privées de Florence (Waquet 2017: 184), ou si leur échange s'est borné à des rencontres dans les bibliothèques devant les manuscrits. Leur dialogue se prolonge toutefois par leur correspondance. Leur goût commun pour les manuscrits donne l'occasion d'échanger sur ce sujet. C'est Jacob Spon qui prend l'initiative de la première lettre, rédigée de Bologne le 25 mai 1675 (Spon 1675: fo 139). Le voyageur y remercie Antonio Magliabechi pour son accueil à Florence. Elle inaugure un échange qui allait durer près de dix ans.

\subsection{Une correspondance érudite entre Lyon et Florence}

La correspondance entre Jacob Spon et Antonio Magliabechi s'étend de 1675 à 1685. L'exil du savant lyonnais en raison de sa foi protestante en septembre 1685, soit quelques semaines avant la révocation de l'édit de Nantes, interrompt leur relation. Le savant quitte Lyon pour rejoindre Zurich et s'y installer, son père Charles Spon (1609-1684) lui ayant légué un droit de bourgeoisie. Mais le médecin ne parvient jamais à destination. Il s'éteint à l'hôpital communal de Vevey sur les rives du lac Léman des conséquences d'une tuberculose chronique. Quarante lettres sont conservées dans les fonds de la bibliothèque municipale de Lyon et de la Biblioteca nazionale centrale de Florence ${ }^{1}$. Toutes les lettres sont rédigées en italien à l'exception de la première lettre de Spon écrite en latin. Mais cette correspondance a été numériquement plus importante si on se fie aux lettres attestées dans les lettres conservées.

1. Lyon, Bibliothèque municipale (BML), ms fonds général 1720 et 1721; Florence, Biblioteca nazionale centrale (BNCF), carteggio magliabechiano, VIII 326; et s. II t. II. 
Tableau 1: Répartition chronologique de l'échange épistolaire entre Jacob Spon et Antonio Magliabechi

\begin{tabular}{|c|c|c|c|}
\hline Année & Reçues par Antonio Magliabechi & Reçues par Jacob Spon & TOTAL \\
\hline 1675 & 1 & & 1 \\
\hline 1676 & 4 & 2 & 6 \\
\hline 1677 & 3 & 7 & 4 \\
\hline 1678 & 3 & 2 & 11 \\
\hline 1679 & 4 & 6 & 6 \\
\hline 1680 & 2 & 1 & 8 \\
\hline 1681 & & & 1 \\
\hline 1682 & 2 & 1 & 2 \\
\hline 1683 & & & 1 \\
\hline 1684 & 1 & 20 & 1 \\
\hline 1685 & 20 & & 40 \\
\hline TOTAL & & & \\
\hline
\end{tabular}

Chaque correspondant est l'auteur de 20 lettres. On remarque un net déséquilibre chronologique. Les trois quarts des lettres sont rédigées entre 1675 et 1680, avec un pic de onze lettres pour l'année 1678. À cette époque, Jacob Spon publie plusieurs ouvrages d'antiquités en s'appuyant sur le matériau ramené de son voyage en Italie et en Grèce. La période 1681-1685 ne compte que cinq lettres échangées. Aucune lettre datée de l'année 1683 n’a été conservée.

Cet échange pèse peu dans les correspondances des deux savants. À ce jour, nous dénombrons 454 lettres écrites ou reçues par Jacob Spon entre 1667 et 1685 pour 86 correspondants. Les 40 lettres représentent à peine 8,81\% de la correspondance recensée. Quant à la correspondance d'Antonio Magliabechi, elle semble être la plus importante de son temps. Rien que pour la correspondance passive, Manuela Doni Garfagnini dénombre 22603 lettres reçues entre 1650 et 1714 (Doni Garfagnini 1988)! Les 20 lettres envoyées par Spon ne représentent que 0,009 \% du total. La correspondance active du bibliothécaire conservée dans différents fonds publics européens n'a pas encore été inventoriée. L'écart quantitatif considérable entre les correspondances des deux épistoliers marque une grande différence de statut au sein de la République des Lettres.

\subsection{Deux savants à la stature sociale et intellectuelle différente}

Antonio Magliabechi est considéré en 1675 comme un intermédiaire majeur dans la circulation de l'information savante en Europe. Fils de cordonnier, il débute comme apprenti orfèvre, puis gravit tous les échelons jusqu'à devenir un familier des Médicis grâce à sa culture bibliographique (Callard 2000 : 91-92). Ses correspondants le qualifient à l'époque de bibliothécaire du grand-duc de Toscane, sans qu'il détienne toutefois cette charge officiellement. Le titre appartient à l'un de ses ennemis, Alessandro Segni (1633-1697). Il bénéficie cependant depuis 1665 d'une charge "officieuse» personnellement liée au Prince et à sa famille (Mirto 2017). Le bibliothécaire s'impose dès sa prise de fonction comme un véritable professionnel du livre (Callard 2000: 95). Antonio Magliabechi joint à ses compétences de bibliothécaire des talents de libraire qui en font l'un des principaux acheteurs de livres à Florence. Il mobilise un impressionnant réseau de contacts, qu'il a constitué dès le début des années 1660, pour 
servir ses maîtres Médicis. Alessandro Segni, davantage courtisan qu'érudit, ne peut rivaliser avec les qualités de Magliabechi. "Jamais homme ne fut plus propre que luy pour cet employ [de bibliothécaire], il a tous les Livres dans sa tête, \& connoît tous les Sçavans de l'Europe ", écrit Spon dans la relation de son voyage en 1677 (Voyage I : 66). Pour retour de ses services, Magliabechi dispose d'avantages que son correspondant, médecin de profession, ne possède pas: un temps entièrement dédié à sa mission, une bourse assez importante pour les achats d'ouvrages et la franchise de port pour sa correspondance (Waquet 2017: 185). Le bibliothécaire fascine ses contemporains par son mode de vie et son physique peu avantageux, en contradiction avec ses qualités intellectuelles (Callard 2000: 86-87). Les 2262 correspondants dénombrés donnent une idée de sa place dans l'espace intellectuel européen (Doni Garfagnini 1988; Van Vugt 2017). Les savants le considèrent comme un des principaux «secrétaires» de la République des Lettres et louent la générosité dont il fait preuve à l'égard des visiteurs érudits de passage à Florence (Waquet 1989 : 396-400; Boutier et Paoli 2005: 399-400) en mettant à leur disposition les manuscrits rares qu'ils recherchent, et ses propres connaissances bibliographiques. La conversation qu'il tient avec ses visiteurs et les informations qu'il reçoit de ses correspondants sont déterminantes dans l'aggiornamento constant de ses connaissances, qui lui confèrent une position surplombante dans la République des Lettres.

Au moment de leur rencontre en mai 1675, Jacob Spon est un jeune médecin cherchant à affirmer son statut de savant antiquaire. Il n'est pourtant pas un inconnu. Il a déjà publié trois livres sur l'Antiquité en 1673 et 1674 . Il entretient un réseau épistolaire sur des sujets liés à l'Antiquité, qui dépasse la sphère d'influence culturelle lyonnaise s'étendant traditionnellement du Mâconnais au sud du Dauphiné et des monts du Forez à la frontière savoyarde. Parmi ses correspondants figurent le jésuite clermontois Gilles Lacarry (1605-1684), le juriste numismate bâlois Sebastian Faesch (16471712) et son grand ami, le médecin numismate en exil Charles Patin (1633-1693), qui lui fournit, depuis Padoue où il réside, des lettres de recommandation pour son séjour italien en 1674-1675. Ses étapes à Rome, Florence, Bologne et Venise lui permettent de se faire connaître des savants et curieux de ces villes et de nouer des relations intellectuelles durables. Toutes les lettres conservées à Lyon envoyées par ses correspondants italiens sont postérieures à ce voyage de formation.

La pérennisation de l'échange épistolaire entre Jacob Spon et Antonio Magliabechi est aussi due aux facilités permises par les relations commerciales que les imprimeurs-libraires lyonnais entretiennent avec Florence. Quelques-uns d'entre eux ont instauré un rapport privilégié avec le bibliothécaire des Médicis. Jugeant que les libraires italiens vendent les livres étrangers à un prix trop élevé, Magliabechi s'épargne leur médiation pour s'adresser directement à leurs collègues français ou allemands afin d'enrichir les bibliothèques de ses maîtres, comme la sienne, des dernières nouveautés à des prix raisonnables. Dès le début des années 1660, Magliabechi a établi une correspondance commerciale avec les imprimeurs-libraires lyonnais Marc-Antoine Ravaud et Guillaume Barbier, Jean Anisson et ses associés, Jean-Antoine II Huguetan et ses fils Marc, Jean Henri et Jean-Antoine III; Philippe Borde et Laurent Arnaud $^{2}$. Le bibliothécaire a instauré pour Florence une relation quasi exclusive avec ces marchands, révélatrice de sa position intellectuelle dominante dans la capitale toscane. La plupart des marchandises, et donc des lettres, transitent par voie maritime entre Marseille et Livourne, jugée par les libraires plus sûre et plus rapide que la voie terrestre. Un intermédiaire, le marchand français Gilles de Gastines, installé dans le port toscan, se charge d'assurer la liaison entre la France et Florence (De Magistris 1985). À Lyon, les lettres d'Antonio Magliabechi sont remises en main propre à Jacob Spon

2. Quelques-unes de ces correspondances ont été étudiées et éditées par des historiens italiens, tels Aniello Fratta (1979), Afonso Mirto $(2005,2008,2011)$ et Salvatore Ussia (1980). 
par les libraires Huguetan (Mirto 2005). Ces plis contiennent une multitude d'informations susceptibles d'être exploitées par les deux correspondants pour leur activité.

\section{2. Échange d'informations et intermédiations}

\subsection{Une correspondance basée sur l'échange d'informations}

Par ses qualités matérielles, la lettre est un des principaux supports de la transmission d'information de la République des Lettres: peu encombrante, donc facile à transporter, elle franchit des distances considérables par le biais de porteurs qui sont souvent eux-mêmes des érudits. La lettre-information est surreprésentée dans les correspondances savantes du XVII ${ }^{\mathrm{e}}$ siècle. L'échange épistolaire entre Jacob Spon et Antonio Magliabechi ne fait pas exception. Ce sont d'abord les renseignements bibliographiques qui sont évoqués: les mentions de nouvelles parutions et d'ouvrages en cours de rédaction ou de publication sont présentes dans quasiment toutes les lettres de notre échange.

Indépendamment de la valeur informationnelle des lettres, Jacob Spon assume clairement sa subordination par rapport à Antonio Magliabechi sur le plan des savoirs. "La riverisco come mio gran patrono et maestro \& la prego de mi dar semper una particella de la sua cientia [sic] se non menti de l'haver tutta intiera» (Spon 1676 3 ). L'antiquaire lyonnais reconnaît le bibliothécaire comme son "patron et maître" détenteur d'une immense "science» à laquelle il espère accéder par un échange de courrier régulier. Cette déférence concerne aussi les informations littéraires que Magliabechi obtient de ses contacts. Spon lui demande de lui fournir des nouvelles d'Italie: "Prego Vostra Signora Illustrissima da favorir mi di nove de la sua favorita in che cosa si tratta de nuovo tra li eruditi in Italia" (Spon 16764). Le bibliothécaire florentin se plie de bonne grâce à cette demande dans chacune de ses lettres en fournissant "qualche novità letterarie». Il relate à son correspondant les querelles scientifiques, comme celle qui intervint autour de la comète du 31 mars 1676 entre l'astronome Geminiano Montanari (16331687) et Pietro Maria Cavina (1637-1690), les controverses religieuses, les morts de savants italiens réputés, etc.

Les premiers échanges de l'antiquaire avec Antonio Magliabechi relatent les péripéties de son voyage: une première lettre est rédigée en août 1675 alors qu'il se rend à Constantinople; une seconde est envoyée de Venise le 18 mai $1676^{5}$ où il annonce son retour sain et sauf en Italie (Spon 1676). Intéressons-nous à cette dernière. Il y informe son correspondant du butin qu'il ramène de Grèce et d'Orient: 400 inscriptions grecques inédites, 600 médailles antiques, et plus de 30 manuscrits dont une vingtaine de grecs comprenant des œuvres de théologiens chrétiens des premiers siècles de notre ère. Parmi les références mentionnées, on compte un manuscrit de Denys l'Aréopagite commenté par Saint Maxime et un manuscrit de l'œuvre de saint Augustin, De Trinitate. Mais peu sûr de la rareté et donc de la valeur de ces manuscrits, Spon demande l'avis du bibliothécaire. La sollicitation de Spon oblige Magliabechi à lui répondre par une lettre datée du 28 août 1676. Le manuscrit de Denys l'Aréopagite n'est pas rare puisqu'il a été imprimé. En revanche, il se réjouit pour lui de l'achat du manuscrit

3. «Je Révère [Sa Seigneurie Illustrissime] comme mon grand patron et maître et je la prie de me donner toujours une parcelle de sa science ou si cela ne vous dérange pas de l'avoir tout entière. »

4. «Je prie Sa Seigneurie Illustrissime de m'accorder de nouveau sa faveur en m'informant des nouveautés venant des érudits d'Italie."

5. Spon débarque à Venise place Saint-Marc le jour de la fête de l'Ascension, le 14 mai. La lettre écrite de Corfou n’a jamais été reçue par le bibliothécaire florentin. 
de saint Augustin, alors peu répandu: "Con mio gran contento veggo che hà comprata la sua versione de' libri di S. Agostino De Trinitate che non è mai stata stampata" (Magliabechi 1676: fo 486).

L'antiquaire lyonnais fournit dans ses lettres des informations provenant de deux réseaux distincts: le premier, parisien et catholique, le renseigne sur l'activité intellectuelle de la capitale de la République des Lettres. Ses correspondants sont pour la plupart médecins, antiquaires ou gens de lettres: François Charpentier (1620-1702), Antoine Galland (1646-1715), Gilles Ménage (16131692), Jean Foy-Vaillant (1632-1706) et surtout le Dijonnais Claude Nicaise (1623-1701), qui se rend occasionnellement dans la capitale. Le second réseau de correspondants de Jacob Spon se distingue par son caractère confessionnel. Calviniste, il nourrit une grande proximité avec Genève en raison de ses attaches familiales, et avec quelques savants établis dans les grands centres intellectuels protestants d'Europe. À Genève, le pasteur Jacques Flournoy (1645-1693), le professeur de langues orientales Michel Turrettini (1646-1721) et l'imprimeur-libraire Jean Hermann Widerholdt (16351683) comptent parmi ses principaux contacts. Aux Provinces-Unies, Jacob Spon noue une relation tardive avec deux correspondants de Magliabechi : le journaliste Pierre Bayle (1647-1706) à Rotterdam et l'antiquaire Gisbert Cuper (1644-1716) à La Haye. En Angleterre, il entretient de bons rapports avec l'historien écossais Gilbert Burnet (1643-1715) qu'il a accueilli à Lyon, et le révérend John Covel (1638-1722), rencontré lors de son voyage au Levant. Spon tire davantage parti du second réseau que du premier pour fournir à son correspondant florentin des informations inédites. Les références aux nouvelles parutions liées à l'Antiquité, à l'histoire ecclésiastique et à la médecine venant des pays protestants sont nombreuses.

Il semble être l'un des premiers à renseigner Antonio Magliabechi de la naissance d'un nouveau périodique à Leipzig en 1682, les Acta eruditorum basé sur le modèle du Journal des sçavans: "Aviso Vostra Signora Illustrissima che in Leipsic s'apparecchiano certi eruditi à darci un Giornale di eruditi a gunsa del Giornale Francese e Italiane, ma lo faranno in Latino" (Spon 1682a ${ }^{7}$ ). Spon est en contact avec le rédacteur du journal Otto Mencke (1644-1707) et avec l'un de ses contributeurs, Friedrich Benedikt Carpzov (1649-1699). L'antiquaire joue quelques mois plus tard un rôle significatif dans la diffusion du journal en France et y publie même des articles médicaux (Laeven 1990: 108-109). Magliabechi noue de son côté une relation épistolaire avec Mencke au début de l'année 1683, lorsqu’on lui remet en main propre le premier volume des Acta eruditorum (Laeven 1990: 111).

Les informations que les savants échangent n'ont toutefois pas vocation à rester confidentielles. Jacob Spon délivre régulièrement à Claude Nicaise les informations transmises par Antonio Magliabechi :

Mr Magliabecchi m'escrit ${ }^{8}$ qu'on luy a envoyé de Naples un livre nouveau intitulé Historia Marsorum libri tres cum eorumdem Episcoporum catalogo Autore Mutio Phoebonio Marso, J. V. D. $4^{\circ}$ Neap[olis] 1678. [...]

$\mathrm{M}^{\mathrm{r}}$ Magliabechi m'ecrit encore qu'il a receu Istoria de Poeti Greci e di que' che in Greca lingua han poetato scritta da Lorenzo Crasso Barone di Pianura. In Napoli, in fo 1678. (Spon 1679b: 323vo)

\footnotetext{
6. «À mon grand contentement, je vois que [Sa Seigneurie Illustrissime] a acheté une version des livres de saint Augustin De Trinitate, qui n’a jamais été imprimée."

7. "J'avise Votre Seigneurie Illustrissime qu’à Leipzig, des érudits s’apprêtent à donner un Journal des érudits sur le modèle des journaux français et italiens, mais qu'ils le feront en latin.»

8. Voir Magliabechi 1679: fo 126.
} 
Cette transmission de l'information par la lettre donne sens à l'existence de ces réseaux savants, car elle est nécessaire aux travaux des lettrés et in fine à l'élaboration collective d'un savoir au nom de l'intérêt public.

\subsection{Deux intermédiaires de la République des Lettres}

En sociologie des réseaux, l'intermédiaire est un agent liant des individus ou des groupes d'individus entre eux (Lemercier 2005: 93). Selon Vincent Lemieux et Mathieu Ouimet, "plus un acteur se trouve dans une position intermédiaire, c'est-à-dire plus il est dans une situation où des acteurs doivent passer par lui pour atteindre d'autres acteurs, plus il aura la capacité de contrôler la circulation de l'information entre ces acteurs» (Lemieux et Ouimet 2004: 24). Autrement dit, la centralité de sa position de «connecteur» lui donne un pouvoir sur les individus qu'il met en relation. Jacob Spon et Antonio Magliabechi ont joué ce rôle d'intermédiaire à des échelles différentes.

Le médecin antiquaire profite à Lyon d'une position géographique favorable entre Paris et l'Italie. Si la ville n'est pas un centre intellectuel majeur, elle a suffisamment d'importance pour que les voyageurs y fassent étape. Spon rencontre régulièrement des savants de passage comme Gregorio Leti (16301701) en 1680 ou Jean Mabillon (1632-1707) en 1682, deux correspondants d'Antonio Magliabechi. Il héberge chez lui par affinité confessionnelle tous les voyageurs érudits anglais et hollandais. Son rôle d'intermédiaire passe aussi par la mise en relation épistolaire de ses correspondants parisiens et du nord de l'Europe avec leurs collègues d'Italie: Charles Patin et Niccolò Bon (1635-1712) à Padoue et Venise, Manfredo Settala (1600-1680) et Antonio Mezzabarba (1645-1697) à Milan, Giuseppe Magnavacca (1639-1724) à Bologne. Cette intermédiation reste cependant faible par rapport au rôle joué par Antonio Magliabechi.

Le recensement des lettres de recommandation paraît être un bon moyen de mesurer la position d'intermédiaire de Jacob Spon dans la République des Lettres. On n’intègre pas ce milieu intellectuel sans cooptation. Ces lettres d'introduction ouvrent au voyageur les portes des cabinets, des bibliothèques et des collections à chaque étape de sa peregrinatio academica. Elles lui permettent de nouer des relations avec les savants qu'il rencontre et d'intégrer de nouveaux circuits épistolaires. L'antiquaire dote ses amis et visiteurs de lettres de recommandation pour l'Italie. Nous en recensons deux adressées à son correspondant florentin. L'abbé Mathieu Pécoil, chanoine de la collégiale Saint-Just à Lyon et grand voyageur, peut ainsi rencontrer à la fin du mois de décembre 1677 Antonio Magliabechi, lequel promet à Spon de lui faire visiter les bibliothèques ducales (Magliabechi 1677: fo $121^{9}$ ). Vers le mois de septembre 1679, Spon recommande également John ou Jean Gailhard, un précepteur anglais d'origine française et calviniste s'intéressant aux antiquités en amateur: "Questa letterina sara per ricommandarli il Signor Gaillard gentilhomo Inglese, mio amico singolare, virtuoso grande e dilettante delle medaille et altre gioie antiche, il qual non vuol mancar di riverir Vostra Signoria Illustrissima passando per Firenza" (Spon $1679 \mathrm{c}^{10}$ ). Gailhard, qui séjourne à Florence au mois d'octobre 1679, paie fort mal la peine qu'a prise son ami d'écrire au bibliothécaire. Il quitte brusquement Antonio Magliabechi, qui ne manque pas de le signaler à Jacob Spon: "Dà quel Signore Inglese mi fù presentata la cortesissima Lettera di Vostra Signoria Illustrissima. Voleva partire quasi subito, onde, con mio dolore non mi fù permesso godere della

9. En réponse à la lettre de Spon datée du 12 octobre (Spon 1677).

10. «Cette petite lettre sera pour recommander [à Votre Seigneurie Illustrissime] le Sieur Gaillard, gentilhomme Anglais, mon ami singulier, [Homme] de grande vertu et dilettante de médailles et d'autres joyaux antiques, lequel ne veut pas manquer de saluer Votre Seigneurie Illustrissime en passant par Florence.» 
sua conversazzione [sic], che per momenti" (Magliabechi 1680a: fo 165 11). Par son départ soudain, peut-être lié à sa charge de précepteur, Gailhard contrevient aux règles de sociabilité savante et prive le bibliothécaire d'une conversation qu'il tient avec tous les érudits qui lui rendent visite.

Par l'étendue de sa correspondance épistolaire, Antonio Magliabechi joue un rôle d'intermédiaire majeur entre les savants italiens et le reste de l'Europe. Son intermédiation ne concerne pas que la mise en liaison d'individus et de réseaux d'individus; elle inclut la transmission des savoirs par la conversation et la lettre. Le bibliothécaire correspond à ce que Christine Berkvens-Stevelinck nomme un intermédiaire culturel, c'est-à-dire "quelqu'un qui transmet des valeurs culturelles d'une mouvance à une autre et qui en est conscient» (Berkvens-Stevelinck et Bots 2005 : 24). La mise en relation entre individus, les échanges matériels, ou l'intercession pour un ami en vue de la publication d'ouvrages sont autant de «valeurs» justifiant ces transmissions.

Antonio Magliabechi a ainsi joué ce rôle pour son correspondant lyonnais. Il le met en relation avec Enrico Noris (1631-1704), professeur d'histoire ecclésiastique et de théologie à l'université de Pise. Nous ne conservons malheureusement aucune lettre de leur liaison épistolaire. Il facilite les contacts avec le jeune antiquaire Paolo Alessandro Maffei (1653-1716), un aristocrate toscan originaire de Volterra. Passionné d'épigraphie, le savant copie à Rome et à Palestrina pour son homologue lyonnais près de 400 inscriptions inédites tirées des recueils du cardinal Francesco Barberini. Son oncle Ugo Maffei, de passage à Lyon en octobre 1679, les remet personnellement à Jacob Spon (Spon 1679d). Il juge ces inscriptions suffisamment précieuses pour en intégrer quelques-unes à ses Miscellanea eruditæ antiquitatis en 1685. Ces recommandations restent néanmoins asymétriques. Jacob Spon ne possède pas le réseau de contacts de son correspondant florentin. Si Mathieu Pécoil est connu de certains savants orientalistes renommés, comme Antoine Galland, le tuteur John Gailhard n'est pas un érudit pouvant rivaliser par sa réputation avec le Père Noris. Cet écart confirme la position secondaire de Spon dans la République des Lettres à la fin des années 1670. Il ne peut jouer à Lyon une intermédiation de premier plan comme son correspondant à Florence, mais peut lui rendre d'appréciables services.

\section{L'expertise savante et les échanges matériels au service de la réputation}

\subsection{Des échanges de services}

La correspondance savante implique la réciprocité des échanges. Qu'ils soient informationnels ou matériels, ces services nécessitent bien souvent un investissement personnel des savants pour satisfaire leurs correspondants. La relation épistolaire entre Jacob Spon et Antonio Magliabechi en donne quelques exemples intéressants sur le plan des savoirs.

L'antiquaire lyonnais a l'occasion de visiter durant ses nombreux voyages des cabinets de curiosités et des collections d'œuvres d'art dont il dresse des listes qu'il publie dans ses ouvrages. Mais ses notes sont souvent loin d'être exhaustives, et, soucieux de donner des renseignements de qualité au public, il n'hésite pas à solliciter l'aide de ses amis pour les compléter. Son premier ouvrage traitant de l'Antiquité, Recherche des antiquités et curiosités de la ville de Lyon (1673), contient trois listes: une des curieux lyonnais, que Spon connaît bien et qu'il a dressée lui-même, une autre des curieux parisiens, fournie par l'antiquaire Jean Foy-Vaillant, et une troisième de "plusieurs curieux et antiquaires, dans

11. «De ce seigneur Anglais, je reçus la très courtoise lettre de Votre Seigneurie Illustrissime. Il voulait partir presque immédiatement, de sorte que je n’ai pu profiter de sa conversation que pour un moment.» 
d'autres villes de l'Europe», dont on ignore l'origine, mais qui est probablement due à l'apport de plusieurs de ses contacts (Spon 1673: 204-224).

Au mois d'avril 1680, Jacob Spon est sur le point d'imprimer la relation d'un voyage d'Italie rédigée par l'avocat protestant Jean Huguetan (1599-1671), mais il souhaite l'enrichir d'une liste des principaux curieux et savants italiens pour satisfaire un lectorat essentiellement français. Il demande alors l'aide de Magliabechi pour lui fournir une liste complète d'érudits:

Devo infinitamente a Vostra Signora Illustrissima delle novita literarie che di tempo in tempo m'a degnato mandar, e cognoscendo la sua prontezza a bearmi [sic] del suo favore, la prego ancora d'ajutarmi in un aggiunta che voglio far a un viaggio d'Italia che si deve presto stampar qua: cioe di mandar mi quanto in una lettera indirizzata diretto per la posta à Mr. Spon fils à Lyon una nota di quanti si ricordara Eruditi, professori celebri, curiose cioe virtuosi e artefici insegni d'Italia, col nome, cognome, patria, cose in che eccellano e principali opere in poche parole, dico di persone viventi. (Spon $1680^{12}$ )

Magliabechi lui répond trois semaines plus tard dans une longue lettre où il mentionne seulement les curieux et savants romains (Magliabechi 1680b: fo 162). La liste est incomplète et il s'en explique dans une lettre rédigée au mois de septembre 1680: il a égaré le reste de ses notes dans le fatras de ses livres (Magliabechi 1680c: fo 100). Il demande alors à Spon de lui renvoyer la liste des savants romains pour éviter les redites dans le nouvel inventaire qu'il s'apprête à rédiger. La demande de Magliabechi demeure sans suite. Spon publie le Voyage d'Italie curieux et nouveau de Jean Huguetan en décembre 1680 avec la liste romaine fournie par le bibliothécaire, et donne une seconde liste de curieux italiens en s'aidant des renseignements fournis par son ami Gregorio Leti dans un de ses ouvrages L'Italia regnante $\left(1675-1676^{13}\right)$.

Dans le sens inverse, Antonio Magliabechi recourt aux services de Jacob Spon pour corriger et veiller à l'impression d'un livre rédigé par un de ses amis:

Un mio grande amico, ed alquale hò molte obbligazzioni, mi hà pregato a fare stampare un Opuscolo in Lione, sapendo ch'io hò conoscenza di alcuni Mercanti di Libri. Io hò mandato li Opuscolo manoscritto, al cortesissimo, ed onoratissimo Signore Huguetan, perche mi onori di farlo stampare a mie spese. Per essere in quarto e stampato stimo che verrà sette, o otto fogli, poiche non si vuole in carattere grande, mà ordinario [...] ed hò scritto al Signore Huguetan che mi faccio li onore di farne stampare cinquecento [...] Perche è un Opuscolo critico, nel quale è necessarissima la correzzione al maggior segno mai possibile, sono a supplicarla, che si voglia degnare di prendersi questo incommodo ella, di badare alla stampa. Ben so, che

12. «Je dois infiniment à Votre Seigneurie illustrissime des nouveautés littéraires que de temps en temps vous daignez m'envoyer, et sachant la promptitude que vous avez à me combler de faveurs, je la prie encore de m’aider pour un supplément que je veux faire à un voyage d'Italie qui doit être imprimé rapidement; c'est de m'envoyer dans une lettre qui me sera directement adressée par la poste à Mr. Spon fils à Lyon une liste des érudits, professeurs célèbres, curieux dont vous vous rappellerez c'est-à-dire des vertueux, et des meilleurs artisans d'Italie avec leur nom, prénom, patrie, et de toutes les choses dans lesquelles ils excellent et leurs principales œuvres en quelques phrases, je parle des personnes vivantes [actuellement].»

13. Federica Missere Fontana (2001: 242-243) indique que Magliabechi a fourni à Leti une liste de savants bolognais rédigée à la fin de l'année 1674 par le banquier numismate Francesco Lotti. Cette histoire met le bibliothécaire florentin dans une position d'intermédiaire inconfortable, car Leti a publié la liste à l'insu de Lotti, qui n’a jamais été informé de la suite qui serait donnée à son texte. Le livre de Leti a été mal reçu du public dès sa sortie. 
Non si danno a suoi, simili impacci mà la necessita mi sforza a ciò, non avendo costà Padrone, o Amico, che possa far questo. (Magliabechi 1680c: fo $100^{14}$ )

L'antiquaire est sollicité non seulement parce qu'il habite Lyon et peut corriger les épreuves sortant des presses, mais aussi parce qu'il est compétent sur le plan théologique et historique pour effectuer une relecture de ce petit ouvrage critique. L'ami qu'Antonio Magliabechi ne nomme pas est le Père Noris. L'ouvrage prend place dans une violente querelle théologique opposant le professeur de théologie pisan au franciscain portugais Francisco de Macedo (1596-1681), enseignant à l'université de Padoue. Ces religieux érudits s'affrontent par ouvrages interposés depuis 1673 sur saint Augustin et la question de la grâce avec une virulence rare qui indigne la République des Lettres. Le bibliothécaire florentin connaît bien Noris et Macedo. Le premier doit sa chaire de théologie à l'intercession de Magliabechi auprès du grand-duc de Toscane Côme III.

Enrico Noris prend le pseudonyme de Fulgence La Fosse pour répondre au franciscain dans cet ouvrage intitulé Somnia quinquaginta fr. Macedo in itinerario S. Augustini post baptismum Mediolano Romam (1681). Spon a bien corrigé les épreuves et Jean-Antoine II Huguetan s'est chargé de l'impression en décembre 1680 aux frais de Magliabechi. En soutenant la publication de ce livre, le bibliothécaire prend discrètement parti pour son ami dans la controverse contre Macedo. Cinquante exemplaires sont remis à Jacob Spon par Huguetan sur ordre de Magliabechi, dont la moitié est envoyée ultérieurement à Jean Mabillon à Paris à qui l'ouvrage est dédié (Mirto 2005: 149). Mais ce sont deux exemplaires que l'antiquaire fait d'abord parvenir anonymement au savant bénédictin en mars 1681 par l'intermédiaire de Claude Nicaise. "J'ay joint [...] trois somnifuges dont vous estes prié de la part du P. Fossé qui en est l'auteur, d'en faire tenir un couple au R. P. Mabillon sans qu'il sache s'il se peut d'où cela vient»(Spon 1681: fo $\left.242 v^{\circ}\right)$.

\subsection{Les échanges matériels: les dons d'ouvrages entre connaissances et reconnaissances}

Derrière le partage désintéressé des connaissances pointe souvent chez les savants le désir de reconnaissance. Ils déploient des «stratégies relationnelles" où les interactions diffèrent en fonction du but à atteindre. Mais méfions-nous de l'usage de cette notion: cela relève davantage d'occasions saisies que d'un plan mûrement réfléchi (Recous 2016: 110). Les dons matériels sont un des moyens mobilisés par les savants pour favoriser leurs intérêts. Il s'agit d'une pratique d'échange traditionnelle au sein de la République des Lettres qui entretient estime et amitié.

Jacob Spon a recours aux dons de manuscrits et de livres parmi lesquels on compte ses propres publications. Ces envois réguliers sont utilisés pour légitimer sa position de savant auprès de ses correspondants. Antonio Magliabechi est gratifié d'un exemplaire de chacun de ses ouvrages à partir de 1676. Les livres d'érudition sont généralement imprimés aux frais de l'auteur et sont destinés à un

14. «Un de mes grand amis, à qui j'ai beaucoup d'obligations, m’a demandé de faire imprimer un petit livre à Lyon, sachant que j'y connais quelques marchands libraires. J'ai envoyé l'œuvre manuscrite au très courtois et très honorable Sieur Huguetan, parce qu'il m’honore de le faire imprimer à mes dépens. Étant imprimé in quarto, j'estime qu'il fera sept ou huit feuilles imprimées parce qu'on ne veut pas de grands caractères mais des caractères ordinaires [...]. J'ai écrit au Sieur Huguetan que je me faisais un honneur de le faire imprimer à 500 exemplaires [...]. Puisque c'est un ouvrage critique dans lequel la correction est absolument nécessaire au premier signe possible, je viens supplier [Votre Seigneurie] de daigner prendre en charge l'incommodité de veiller à l'impression, même si nous ne pouvons vous donner de services similaires, mais la nécessité m’y contraint, n'ayant pas de maître ou d'ami pouvant le faire.» 
public restreint. Ils sont donc coûteux à fabriquer et difficiles à écouler. Spon est bien conscient de la position centrale du bibliothécaire dans l'espace savant européen. Son avis peut décider du succès ou de l'échec d'un ouvrage. Le médecin-antiquaire compte donc sur les faveurs de son correspondant pour toucher un public lettré européen susceptible de les acheter et de les recommander à leurs amis.

Sans être un spécialiste de l'Antiquité, Antonio Magliabechi estime les travaux de Jacob Spon. Il facilite le débit du Voyage d'Italie, de Dalmatie, de Grèce et du Levant de Spon en Italie: "Non hò mancato di dare avviso di questa eruditissima Opera a tutti i miei Amici eruditi d'Italia", lui écrit-il en mars 1678 (Magliabechi 1678: fo $159^{15}$ ). Il juge très favorablement la première section des Miscellanea eruditx antiquitatis publiée en 1679: "L'opera è eruditissima, e stampata come merita, con la maggior nobilità che mai si possa per la bellezza delle figure, della carta, del carattere, e di ogni cosa. Come sarà finita, riescirà uno de maggiori ornamenti del nostro Secolo questa Opera senza alcun dubbio» (Magliabechi 1680a: fo $165^{16}$ ).

Contrairement à Spon, le bibliothécaire n'est pas un auteur affirmé, mais il utilise les mêmes procédés que son correspondant pour soutenir sa réputation comme celle de ses amis. En décembre 1678, l'antiquaire lyonnais reçoit de Florence plusieurs livres envoyés par Magliabechi: un exemplaire de la Biblioteca volante (1677) du médecin-bibliographe Giovanni Cinelli Calvoli (1625-1706), à laquelle le bibliothécaire florentin aurait pris part comme auteur (Viola 2017 : 166-168). L'ouvrage cite Jacob Spon et un ouvrage portant sur les instruments musicaux antiques, De tibiis veterum (1677), imprimé à Rome par un de ses correspondants, le médecin antiquaire danois Caspar II Bartholin (1655-1738).

Comme c'est le cas avec l'opuscule critique de Noris contre Macedo, le bibliothécaire participe à l'édition de textes. À la fin de l'année 1681, Antonio Magliabechi envoie à son correspondant un exemplaire d'un livre en latin, Hodoeporicon, rédigé par l'humaniste camaldule Ambrogio Traversari (1386-1439), qui relate sa tournée d'inspection dans les monastères de son ordre en Italie. Le texte est édité par un des amis du bibliothécaire, Niccolo Francesco Bartolini da Barga (Traversari 1681). L'Hodoeporicon est à l'origine un manuscrit conservé à la Laurenziana, que Magliabechi a mis au jour en 1677, après avoir obtenu l'autorisation du grand-duc Côme III de le copier et de l'imprimer. L'ouvrage débute par neuf pages d'éloges de Magliabechi, qui est présenté comme le véritable éditeur (Boutier 2017: 364). Le bibliothécaire s'est beaucoup investi dans la mise au point de cette édition, attendue des érudits, et dont il assure la dépense. Il est donc normal qu'il en fasse la promotion auprès de ses correspondants, susceptibles de le promouvoir à leur tour auprès de leurs réseaux.

Antonio Magliabechi use des mêmes méthodes que Jacob Spon pour s'affirmer en tant que savant, c'est-à-dire en tant que praticien et non plus seulement intermédiaire des savoirs qu'il assume par sa profession de bibliothécaire. Mais, contrairement à Spon, qui bénéficie du travail soigné des imprimeurs lyonnais, Magliabechi pâtit de la médiocre qualité de l'impression florentine de son ouvrage, d'autant plus que l'édition ne possède pas de commentaire critique (Boutier 2017: 366). Les savants trouvent donc un intérêt limité à l'achat de ce livre de 72 pages. Spon ne souhaite pas le conserver et propose d'offrir son exemplaire à l'abbé Claude Nicaise quelques semaines plus tard (Spon 1682b: fo $267 \mathrm{v}^{\circ}$ ), tout comme l'exemplaire de la Biblioteca volante de Cinelli, qu'il juge «absolument inutile» pour ses travaux d'érudition (Spon 1679a: fo 218ro $)$.

15. "Je n'ai pas manqué d'augmenter le crédit de cette œuvre très érudite à tous mes amis érudits d'Italie."

16. «L'œuvre est très érudite et imprimée comme elle le mérite, avec la plus grande noblesse que l'on puisse attendre pour la beauté des figures, du papier, des caractères et de toute chose. Quand elle sera achevée, cette œuvre sera sans aucun doute l'un des plus grands ornements de notre siècle." 
Ces quelques pages ont ébauché les principales caractéristiques de la correspondance entre Jacob Spon et Antonio Magliabechi. Correspondance qui dessine l'une des configurations possibles de l'échange d'informations entre deux acteurs de la République des Lettres. En dépit de leurs différences sociales et confessionnelles, le bibliothécaire florentin et l'antiquaire lyonnais échangent des nouvelles et des services essentiels à leurs activités respectives. On repère en filigrane, derrière le respect scrupuleux de la communication savante imposant la réciprocité, les processus de légitimation et de médiatisation déployés pour la reconnaissance du statut intellectuel auquel chacun aspire. Spon, marginalisé à Lyon, désire accroître sa réputation de savant antiquaire en Europe en soignant sa relation avec le bibliothécaire. Magliabechi trouve en son interlocuteur lyonnais un informateur fiable et un agent favorisant la promotion et l'impression des ouvrages de ses amis comme des siens en tant qu'éditeur. Il ne faudrait toutefois pas isoler cet échange épistolaire des réseaux savants où il s'insère entre la France et l'Italie. Une étude à plus grande échelle de leurs interactions de part et d'autre des Alpes permettrait de recontextualiser la place de la correspondance entre Jacob Spon et Antonio Magliabechi dans les débats autour de l'érudition historique.

\section{Références bibliographiques}

Bartholin, C. T. 1677. De Tibiis veterum \& earum antiquo usu libri tres. Rome: B. Carrara.

Berkvens-Stevelinck, C. et H. Bots. 2005. "Introduction». Dans Les grands intermédiaires culturels de la République des Lettres: Études de réseaux de correspondances du xvi au xviiie siècles, sous la direction de C. Berkvens-Stevelinck, H. Bots et J. Häseler. Paris: Honoré Champion.

Boutier, J. et M. P. Paoli. 2005. «Letterati cittadini e principi filosofi. I milieux intellettuali fiorentini tra Cinque e Settecento". Dans Naples, Rome, Florence: Une histoire comparée des milieux intellectuels italiens (xviiexviiie siècles), sous la direction de J. Boutier, B. Marin et A. Romano. Rome: École française de Rome: 331-403.

Boutier, J., M. P. Paoli et C. Viola, dir. 2017. Antonio Magliabechi nell'Europa dei saperi. Pise: Edizioni della Normale.

Boutier, J. 2017. "Le "petit monde" parisien de Magliabechi». Dans Antonio Magliabechi nell'Europa dei saperi, sous la direction de J. Boutier, M. P. Paoli et C. Viola. Pise: Edizioni della Normale: 333-378.

Callard, C. 2000. «Diogène au service des princes: Antonio Magliabechi à la cour de Toscane (1633-1714)». Histoire, Économie et Société 19 (1): 85-103. DOI: 10.3406/hes.2000.2108.

Cinelli Calvoli, G. 1677. Della Biblioteca volante di Giovanni Cinelli scanzia prima. Florence: G. A. Bonardi.

De Magistris, R. 1985. «Il mercante e il bibliotecario : la circolazione del libro nelle lettere di Gilles de Gastines a Magliabecchi (1661-1709)». Biblioteche oggi 3: 93-113.

Doni Garfagnini, M. 1988. Lettere e carte Magliabechi. Inventario cronologico. Rome: Istituto storico italiano per l'età moderna e contemporanea.

Étienne, R. et J.-C. Mossière, dir. 1993. Jacob Spon: Un humaniste lyonnais du xviie siècle. Paris: Diffusion de Boccard.

Fratta, A. 1979. "L'attività degli editori Anisson di Lione nel carteggio con Antonio Magliabechi (1669-1708)». Sociologia della Letteratura 3: 115-129.

Huguetan, J. 1681. Voyage d'Italie curieux et nouveau: enrichi de deux listes, l'une de tous les curieux et de toutes les principales curiositez de Rome, et l'autre de la pluspart des sçavans, curieux et ouvriers excellens de toute l'Italie à présent vivans. Lyon: Thomas Amaulry. 
Laeven, H. 1990. The "Acta eruditorum" under the Eitorship of Otto Mencke (1644-1707): The History of an International Learned Journal between 1682 and 1707. Amsterdam: APA-Holland University Press.

Lemercier, C. 2005. «Analyse de réseaux et histoire». Revue d'histoire moderne et contemporaine 52 (2) : 88-112. DOI : $10,3917 /$ rhmc.522.0088.

Lemieux, V. et M. Ouimet. 2004. L'analyse structurale des réseaux sociaux. Québec et Bruxelles: Les Presses de l'Université de Laval et De Boeck Université.

Leti, G. 1675-1676. L'Italia regnante ò vero nova descritione dello stato presente di tutti prencipati, e republiche d'Italia, 4 vol. Genève: G. et P. de La Pietra.

Magliabechi, A. 1676. Lettre à Jacob Spon (28 août). Ms fonds général 1720. Bibliothèque municipale: Lyon.

Magliabechi, A. 1677. Lettre à Jacob Spon (30 décembre). Ms fonds général 1721. Bibliothèque municipale: Lyon.

Magliabechi, A. 1678. Lettre à Jacob Spon [mars]. Ms fonds général 1721. Bibliothèque municipale: Lyon.

Magliabechi, A. 1679. Lettre à Jacob Spon (28 mai). Ms fonds général 1721. Bibliothèque municipale: Lyon.

Magliabechi, A. 1680 a. Lettre à Jacob Spon (1 ${ }^{\text {er }}$ janvier). Ms fonds général 1721. Bibliothèque municipale: Lyon.

Magliabechi, A. 1680b. Lettre à Jacob Spon (17 mai). Ms fonds général 1721. Bibliothèque municipale: Lyon.

Magliabechi, A. 1680c. Lettre à Jacob Spon (14 septembre). Ms fonds général 1721. Bibliothèque municipale: Lyon.

Mirto, A. 2005. Il carteggio degli Huguetan con Antonio Magliabechi e la corte medicea: Ascesa e declino di un'impresa editoriale nell'Europa seisettecentesca. Soveria Mannelli (Catanzaro) : Rubbettino.

Mirto, A. 2008. «Lettere di Borde, Arnaud, Rigaud e associati lionesi a Carlo Roberto Dati, Antonio Magliabechi, Leopoldo e Cosimo III de' Medici». Culture del testo e del documento 26: 5-23.

Mirto, A. 2011. Lettere degli Anisson Poseu e Rigaud, librai lionesi ad Antonio Magliabechi e alla corte medicea. Naples: Scriptaweb.

Mirto, A. 2017. «Al servizio dei principi. Antonio Magliabechi e i Medici». Dans Antonio Magliabechi nell'Europa dei saperi, sous la direction de J. Boutier, M. P. Paoli et C. Viola. Pise: Edizioni della Normale: 91-131.

Missere Fontana, F. 2001. «Raccolte numismatiche e scambi antiquari a Bologna fra Quattrocento e Seicento. Parte II». Bollettino di Numismatica 36-37: 207-318.

Noris, E. 1681. Somnia quinquaginta fr. Macedo in itinerario S. Augustini post baptismum Mediolano Romam, excutiebat levi brachio P. Fulgentius Fosseus Augustinianus ad reverendissimum Johannem Mabillon. Lugduni Batavorum [Lyon] : s. e. [Jean-Antoine II Huguetan].

Rebaudo, L. 1998. «Il viaggio in Italia e il metodo antiquario di Jacob Spon». Dans Annali della Scuola Normale Superiore di Pisa. Classe di Lettere e Filosofia. Quaderni 6 (2): Dell'antiquaria e dei suoi metodi: Atti delle giornate di studio, sous la direction de E. Vaiani: 111-138.

Recous, N. 2016. «S'intégrer dans la République des Lettres. Le cas de Nicolas Fatio de Duillier (1681-1688)». Revue historique 677: 83-112. DOI: 10,3917/licla1.088.0035.

Spon, J. 1673. Recherche des antiquités et curiosités de la ville de Lyon, ancienne colonie des Romains \& capitale de la Gaule celtique. Avec un Mémoire des principaux antiquaires \& curieux de l'Europe. Lyon: Jaques Faeton.

Spon, J. 1675. Lettre à Antonio Magliabechi (25 mai). Carteggio Magliabechiano, s. II, t. II. Biblioteca nazionale centrale: Florence.

Spon, J. 1676. Lettre à Antonio Magliabechi (18 mai). Carteggio Magliabechiano, VIII : 326, lettre ${ }^{\circ} 1$. Biblioteca nazionale centrale: Florence.

Spon, J. 1677. Lettre à Antonio Magliabechi (12 octobre). Carteggio Magliabechiano, VIII: 326, lettre ${ }^{\circ} 7$. Biblioteca nazionale centrale: Florence.

Spon, J. 1678. Voyage d'Italie, de Dalmatie, de Grèce et du Levant, fait aux années 1675 \& 1676 par Jacob Spon docteur medecin aggregé à Lyon, \& George Wheler gentilhomme anglois, 3 vol. Lyon: Antoine Cellier le fils.

Spon, J. 1679a. Lettre à Claude Nicaise (20 janvier). Ms fr 9360. Bibliothèque nationale de France: Paris. 
Spon, J. 1679b. Lettre à Claude Nicaise (juin). Ms fr 9360. Bibliothèque nationale de France: Paris.

Spon, J. 1679c. Lettre à Antonio Magliabechi [septembre]. Carteggio Magliabechiano, s. II, t. II, lettre no 148. Biblioteca nazionale centrale: Florence.

Spon, J. 1679d. Lettre à Antonio Magliabechi (10 octobre). Carteggio Magliabechiano, VIII : 326, lettre nº 14. Biblioteca nazionale centrale: Florence.

Spon, J. 1680. Lettre à Antonio Magliabechi (26 avril). Carteggio Magliabechiano, VIII: 326, lettre no 17. Biblioteca nazionale centrale: Florence.

Spon, J. 1681. Lettre à Claude Nicaise (21 février). Ms fr 9360. Bibliothèque nationale de France: Paris.

Spon, J. 1685. Miscellanea erudite antiquitatis, in quibus marmora, statue, musiva, toreumata, gemma, numismata, Grutero, Ursino, Boissardo, Reinesio, aliisque antiquorum monumentorum collectoribus ignota, \& hucusque inedita referuntur ac illustrantur: curâ \& studio Jacobi Sponii. Lyon: T. Amaulry, sumptibus auctoris.

Traversari, A. 1681. Beati Ambrosii abbatis generalis Camaldulensis Hodoeporicon. Florence: S. et G. D. Marescandoli.

Ussia, S. 1980. Carteggio Magliabechi: Lettere di Borde, Arnaud e associati lionesi ad Antonio Magliabechi (16611700). Florence: L. S. Olschki.

Van Vugt, I. 2017. "Geografia e storia di una rete epistolare. Contatti e mediazioni nell'epistolario di Magliabechi». Dans Antonio Magliabechi nell'Europa dei saperi, sous la direction de J. Boutier, M. P. Paoli et C. Viola. Pise: Edizioni della Normale: 259-292.

Viola, C. 2017. «Magliabechi “autore”». Dans Antonio Magliabechi nell'Europa dei saperi, sous la direction de J. Boutier, M. P. Paoli et C. Viola. Pise: Edizioni della Normale: 143-177.

Waquet, F. 1982. "Antonio Magliabechi: nouvelles interprétations, nouveaux problèmes». Nouvelles de la République des Lettres 1: 173-188.

Waquet, F. 1989. Le modèle français et l'Italie savante: Conscience de soi et perception de l'autre dans la République des lettres, 1660-1750. Rome: École française de Rome.

Waquet, F. 2017. "“Faticare a benefizio della letteraria Repubblica”: Magliabechi et la communication du savoir». Dans Antonio Magliabechi nell'Europa dei saperi, sous la direction de J. Boutier, M. P. Paoli et C. Viola. Pise: Edizioni della Normale: 181-199. 